\title{
Precursory studies on the orchid flora of Myanmar with one new species and thirty eight new distribution records
}

\author{
Hubert Kurzweil ${ }^{1 *}$ and Paul Ormerod ${ }^{2}$ \\ 'Singapore Botanic Gardens, 1 Cluny Road, Singapore - 259569. \\ 2P.O. Box 8210, Cairns 4870, Queensland, Australia. \\ *E-mail: hubert_kurzweil@nparks.gov.sg
}

\begin{abstract}
The new species Calanthe kermodei is described here. In addition, thirty eight new distribution records in the genera Bulbophyllum, Bulleyia, Calanthe, Chrysoglossum, Cleisostoma, Crepidium, Dendrobium, Galearis, Goodyera, Habenaria, Herminium, Liparis, Malaxis, Neottia, Nephelaphyllum, Oberonia, Oreorchis, Pogonia, Ponerorchis and Zeuxine are reported. All of the newly recorded species are found in adjacent countries, and their occurrence in Myanmar is therefore not surprising.
\end{abstract}

Keywords: Calanthe kermodei, Myanmar, new records, Orchidaceae

\section{Introduction}

The orchid flora of Myanmar is very diverse but is by far the most poorly known in mainland Southeast Asia. In the last few years a number of new species and new distribution records have been published (Liu et al., 2018), but a comprehensive floristic account is not available as yet. In preparation of an annotated checklist of all Orchidaceae reported from Myanmar so far (Ormerod et al., in prep.) one new species is described and thirty eight new distribution records are reported here.

Calanthe kermodei Ormerod \& Kurzweil, sp. nov.

Fig. 1

Type: MYANMAR, Kachin State, southeastern part of Myitkyina district, Laikam to Fengshuiling road, $2440 \mathrm{~m}, 22.4 .1938$, C.W.D. Kermode 17210 (Holotype, AMES!; Isotype, K!).

Similar to C. chloroleuca Lindl. but the spur is shorter (4-6 mm vs. 8-12 mm), lip ecarinate (vs. keeled), epichile unguiculate (vs. subsessile), reniform and entire (vs. obcordate and bilobulate). Also close to $C$. griffithii Lindl. but the leaves are in early stages of development during flowering (vs. well-developed at anthesis), floral bracts 9-18 $\mathrm{mm}$ long (vs. 4-5 $\mathrm{mm}$ long), and the reniform and apically acute lip midlobe with a callus consisting of a low median ridge (vs. emarginate and minutely apiculate with prominent erect and tooth-like triangular callus).
Terrestrial herbs, $47-58 \mathrm{~cm}$ tall. Cataphylls to $16 \mathrm{~cm}$ long. Pseudobulbs not seen, 2-3-leaved. Leaves in early stages of development, appear to be narrowly elliptic, plicate, subglabrous, subacuminate. Inflorescences erect, stout, $39-55 \mathrm{~cm}$ long, hairy; peduncle $19-25 \mathrm{~cm}$ long, $0.5-0.7 \mathrm{~cm}$ thick; sterile bract 1, suberect, $2 \mathrm{~cm}$ long, subglabrous; rachis sublaxly to laxly 20 or more-flowered, $15-24 \mathrm{~cm}$ long; floral bracts ovate-lanceolate, acute, 9-18 $\times c$. $4 \mathrm{~mm}$, sparsely hairy. Flowers green, lip yellowgreen. Pedicel and ovary narrowly cylindricclavate, finely shortly pubescent, c. $22 \mathrm{~mm}$ long. Median sepal broadly oblong, acute, 5-6-veined, c. $19.0 \times 7.9 \mathrm{~mm}$, outside sparsely hairy. Lateral sepals broadly oblong-lanceolate, acute, 5 -veined, 18.5-19.0 × 7-7.9 mm, outside sparsely hairy. Petals oblong-lanceolate, acute, 3-5-veined, 16-17 $\times$ c. $5 \mathrm{~mm}$, outside glabrous or subglabrous. Lip spurred, trilobed, joined to column for c. $6 \mathrm{~mm}$, glabrous, without prominent ornaments; spur narrowly conical, obtuse, weakly curved, 4-6 mm long, sparsely hairy; side lobes obliquely cuneate, obtuse, 7-8 × 4-4.5 mm; epichile unguiculate, blade subquadrate-reniform with acute median projection at its tip, callus as a low median ridge, c. $8.0 \times 6.2 \mathrm{~mm}$, unguis $3.5-4.0 \times$ c. $2.5 \mathrm{~mm}$. Column $9 \mathrm{~mm}$ long (from column base to tips of column wings); column wings deltate, subacute, free from lip side lobes.

Flowering: April. 


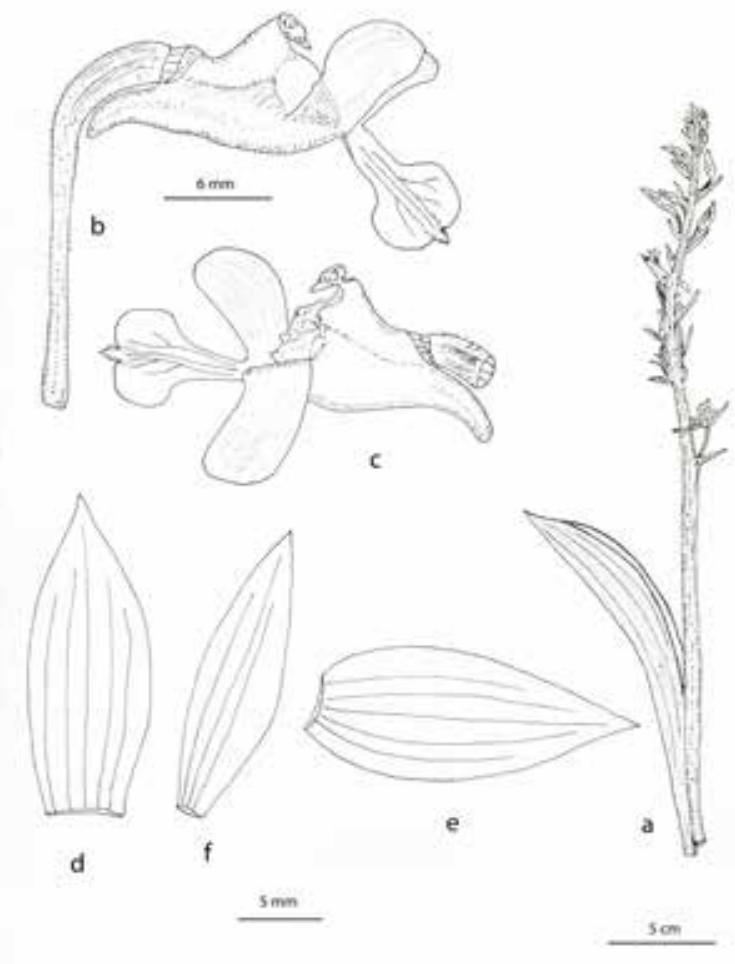

Fig. 1. Calanthe kermodei Ormerod \& Kurzweil.: a. Leaf and inflorescence; $\mathbf{b}$-c, Flower, sepals and petals removed; d. Dorsal sepal; e. Lateral sepal; $\boldsymbol{f}$. Petal. Drawn from the holotype.

Distribution $\mathcal{E}$ habitat: Currently known only from the type locality in Myitkyina District, Kachin State, Myanmar. According to the collector's notes, the species was collected at an elevation of about $2440 \mathrm{~m}$. No habitat notes are given on the herbarium label.

Etymology: The new species is named after the collector of the type specimen, C.W.D. Kermode.

\section{New records}

The list of new distribution records in Myanmar is given below. Detailed localities are not given for conservation reasons, in order not to facilitate the collecting of the plants. Unless otherwise indicated all specimens were identified by one of us.

Bulbophyllum candidum (Lindl.) Hook.f., Fl. Brit. India 5: 770. 1890. Ione candida Lindl., Fol. Orchid. 2: 3. 1853. Phyllorkis candida (Hook.f.) Kuntze, Revis. Gen. Pl. 2: 677. 1891. Sunipia candida (Lindl.) P.F. Hunt, Kew Bull. 26: 183. 1971.

Type: INDIA, Khasia Hills, 1525-1830 m, Hooker E Thompson 70 (Holotype, K-LINDL!).
Epiphytic herbs, pseudobulbs spaced at intervals of 1-2 cm on a creeping rhizome, ovoid, c. $1.5 \times$ $0.7 \mathrm{~cm}$, one-leaved. Leaves linear to narrowly oblong, shortly petiolate, 3-6.5 × 0.4-0.9 cm, obtuse, leathery. Inflorescences 1 or 2, erect, to 9 $\mathrm{cm}$ long; peduncle slender, sheathed; racemes 2.5-3.5 cm long, with 5 to 8 laxly arranged flowers; floral bracts lanceolate, 4-7 mm long, acuminate. Flowers thinly textured; sepals and petals white or greenish white, lip yellow. Median sepal ovatelanceolate, 5-6 × 1.2-1.5 mm, acute or acuminate; lateral sepals similar to the median sepal. Petals oblong-ovate, $c .3 \times 1.7 \mathrm{~mm}$, acute, margins erose. Lip ovate-lanceolate, 5-6 × 1.5-2 mm, obtuse, keeled, erose-lacerate on the margins. Column $c$. $1 \mathrm{~mm}$ long; column-foot c. $1 \mathrm{~mm}$ long. Pedicel and ovary 3-7 mm long.

Distribution: North-eastern India, Bhutan, northern Myanmar (Kachin State), southwestern China (Xizang, Yunnan).

Specimens examined: MYANMAR, Kachin State: Putao Township, 21.3.2009, Kurzweil \& Saw Lwin KL 2637 (SING, spirit collection).

Bulbophyllum elatum (Hook.f.) J.J. Sm., Bull. Jard. Bot. Buitenzorg, sér. 2, 8: 23. 1912. Cirrhopetalum elatum Hook.f., Fl. Brit. India 5: 775. 1890. Phyllorkis elata (Hook.f.) Kuntze, Revis. Gen. Pl. 2: 677. 1891.

Type: INDIA, Darjeeling, 1525 m, 9.1882, Gamble 10560 (Syntype, K!).

Epiphytic herbs, pseudobulbs spaced at intervals of up to $1 \mathrm{~cm}$ on a stout creeping rhizome, conicalcylindric or elongate-cylindric, c. $3 \mathrm{~cm}$ long, oneleaved. Leaves linear-oblong or oblong, petiolate, 9.5-12 × 1.9-3.1 cm, obtuse, somewhat emarginate. Inflorescences from the pseudobulb base, c. 32 $\mathrm{cm}$ long; peduncle slender, sheathed; racemes subumbellate, several-flowered; floral bracts lanceolate, 5-7 mm long, acute. Flowers dull yellow or yellow-green, with purple spots. Median sepal ovate, to $13 \times 7 \mathrm{~mm}$, subacute, hooded; lateral sepals narrowly oblong, c. $20 \times 4 \mathrm{~mm}$, acute. Petals ovate-triangular, $4-7 \times 2.5-4 \mathrm{~mm}$, acute. Lip ovatelanceolate to oblong, 4-6 × 1.5-2 mm, acute, fleshy, upper surface basally grooved. Column $2-3 \mathrm{~mm}$ long; column-foot 3-4 mm long; stelidia slender and forwards curved. Pedicel and ovary $1.5-2 \mathrm{~cm}$ long.

Distribution: Nepal, North-eastern India, Bhutan, northern Myanmar (Kachin State), south-western China (Xizang, Yunnan), Vietnam.

Specimens examined: MYANMAR, Kachin State: Nogmung Township, 915-1220 m, 14.9.1926, Kingdon-Ward 7408 (K). 
Notes: The collection Kingdon-Ward 7408 was originally identified as Bulbophyllum elatum (probably by Kew Herbarium staff) but differs from type specimens in being leafless, although it is not clear whether this was the natural state or the leaf fell off later. No lip is present in the material but the remainder of the flower structure (including the peculiar column architecture) is typical of this species.

Bulbophyllum intricatum Seidenf., Dansk Bot. Ark. 33 (3): 119. 1979.

Type: THAILAND, Mae Hong Son Province, Pang Mo, Seidenfaden E Smitinand 2521 (Holotype, C).

Epiphytic herbs, pseudobulbs spaced at intervals of 3.5-6 cm on a creeping rhizome, ovoid, $3-6 \mathrm{~cm}$ high, one-leaved. Leaves oblong, shortly petiolate, $10-16 \times 2.5-4.0 \mathrm{~cm}$, obtuse. Inflorescences arching; peduncle sheathed; racemes $c .10 \mathrm{~cm}$ long, with many semi-densely arranged flowers; floral bracts shorter than pedicel and ovary. Flowers yellow or greenish yellow. Median sepal ovate, hooded, 4.6-5.5 mm long, obtuse; lateral sepals oblique, 6-8.4 mm long, connate with each other to form a boat-like structure. Petals very broadly triangular, c. $2.8 \mathrm{~mm}$ long, subacute, broad base decurrent on column-foot. Lip ovate-lanceolate, $4.5-5 \mathrm{~mm}$ long, subacute, with a longitudinal groove. Column c. $3 \mathrm{~mm}$ long; column-foot c. $3 \mathrm{~mm}$ long; stelidia narrowly triangular, erect, as tall as anther.

Distribution: Myanmar (Shan State), Thailand.

Specimens examined: MYANMAR, Shan State: south-western part, $1480 \mathrm{~m}, 15.12 .2014$, Fujikawa, Baba, Watthana E Htike San Soe 101848 (MBK).

Notes: Seidenfaden (1979: 122 \& 135) pointed out that two plants cultivated at Kew and mounted on the type sheet of Bulbophyllum pechei Bull may be referable to this species. As B. pechei is endemic to Myanmar, it is possible that the cultivated plants come from the same country.

Bulbophyllum orientale Seidenf., Dansk Bot. Ark. 33 (3): 138. 1979.

Type: THAILAND, Phitsanulok Province, Thung Salaeng Luang National Park, Salaeng Haeng River, Seidenfaden \& Smitinand 5602 (Holotype, C).

Epiphytic herbs, pseudobulbs spaced at intervals of 4-6 cm on a stout creeping rhizome, ovoid, 2-3 cm high, one-leaved. Leaves oblong, shortly petiolate, $8-30 \times 1.5-3.4 \mathrm{~cm}$, obtuse, leathery. Inflorescences basal, arching; peduncle stout, 5-13 cm long, sheathed; racemes $3-6 \mathrm{~cm}$ long, with many densely arranged flowers; floral bracts ovate-lanceolate, 5-6 mm long, acute. Flowers greenish yellow, with darker markings. Median sepal ovate, c. $6 \times 3 \mathrm{~mm}$, acute; lateral sepals oblique, c. $7.5 \times 4.2 \mathrm{~mm}$, acute, papillose, connate and forming a sac. Petals obliquely triangular, $c$. $2.5 \times 1.8 \mathrm{~mm}$, aristate. Lip ovate-lanceolate, c. $6 \mathrm{~mm}$ long, basally grooved, obtuse, with falcate and irregularly toothed basal auricles c. $1.3 \mathrm{~mm}$ long. Column c. $2 \mathrm{~mm}$ long; column-foot c. $3 \mathrm{~mm}$ long; stelidia subulate, curved forwards and sideways.

Distribution: Myanmar, northern Thailand, Cambodia, Laos, Vietnam, South-western China (Yunnan).

Specimens examined: MYANMAR, without locality, s.d., cult. Glasnevin Botanic Gardens s.n. (K); SBGO 3651 (SING, spirit collection), det. J.J. Vermeulen.

Bulbophyllum rolfei (Kuntze) Seidenf., Dansk Bot. Ark. 33 (3): 149. 1979. Phyllorkis rolfei Kuntze, Revis. Gen. Pl. 2: 676. 1891. Cirrhopetalum parvulum Hook.f., Fl. Brit. India 5: 778. 1890. Bulbophyllum parvulum (Hook.f.) J.J. Sm., Bull. Jard. Bot. Buitenzorg, sér. 2, 8: 27. 1912, nom. illeg.

Type: INDIA, Darjeeling, Griffith 5174 (Holotype, $K !)$.

Epiphytic herbs, pseudobulbs spaced at intervals of $0.1-2.4 \mathrm{~cm}$ on a creeping rhizome, ovoid, to $1.5 \times 0.9 \mathrm{~cm}$, one-leaved. Leaves elliptic-oblong to oblong, shortly petiolate, $1.5-3.5 \times 0.8-1.2$ $\mathrm{cm}$, subacute or obtuse, leathery. Inflorescences basal, 4-5.5 cm long; peduncle slender, sheathed; racemes subumbellate, with 2 to 4 flowers; floral bracts lanceolate, 6-8 $\mathrm{mm}$ long, acuminate. Flowers pendent, yellow to red-purple, lip deep purple. Median sepal oblong-ovate, 5-6 × 1.2-2.0 $\mathrm{mm}$, acute; lateral sepals free, linear-oblong, 10-16 $\times 2-3 \mathrm{~mm}$, acute. Petals elliptic-ovate, c. $4.5 \times 2 \mathrm{~mm}$, obtuse. Lip oblong, 3-4 × 0.5-0.8 mm, subacute, adaxially finely papillose, upper surface grooved. Column 1-1.5 mm long; column-foot c. $2.5 \mathrm{~mm}$ long, curved; stelidia c. $1 \mathrm{~mm}$ long, erect, linear. Pedicel and ovary $4-7 \mathrm{~mm}$ long.

Distribution: Nepal, North-eastern India, Bhutan, northern Myanmar (Kachin State), South-western China (Yunnan).

Specimens examined: MYANMAR, Kachin State, Nogmung Township, 12.2015, Saw Lwin KSL 1080 \& 1089 (RAF).

Bulbophyllum scabratum Rchb.f. in W.G. Walpers, Ann. Bot. Syst. 6: 259. 1861. Cirrhopetalum caespitosum Wall. ex Lindl., Edwards's Bot. Reg. 24 (Misc.): 35. 1838. 
Type: EAST INDIES (likely Northeast India), under cultivation, Duke of Devonshire s.n. (Holotype, K) [fide Kumar et al., 2018: 70].

Bulbophyllum levinei Schltr., Repert. Spec. Nov. Regni Veg. 19: 381. 1924.

Types: CHINA, Guangdong, Lo Fau Shan, $800 \mathrm{~m}$, 8.1917, Levine 1548 (Syntype, B [destroyed]); same date and locality, Merrill 10696 (Syntype, HK [fide Baretto et al., 2011: 568]; Isosyntype, AMES [3 sheets]).

Epiphytic herbs, pseudobulbs adjacent, conicalovoid, $0.5-1.3 \times 0.2-0.6 \mathrm{~cm}$, one-leaved. Leaves narrowly oblong, shortly petiolate, $3-5(-9) \times$ $0.5-1.3 \mathrm{~cm}$, subacute, leathery. Inflorescences from base of pseudobulb, shorter to slightly longer than leaves; peduncle slender, 3-5 cm long; racemes subumbellate, with 2 to 6 flowers; floral bracts lanceolate, 2-4 mm long, acute. Flowers yellow or white with purple tinge. Median sepal ovate-lanceolate, $3-5 \times 1.5-2.0 \mathrm{~mm}$, acute, often denticulate at the margins; lateral sepals free, narrowly lanceolate, c. $11 \times 3.0 \mathrm{~mm}$, acuminatecaudate. Petals ovate-lanceolate, $2-3.5 \times 1-2 \mathrm{~mm}$, acute, margins denticulate. Lip oblong-lanceolate, 2-2.5 mm long, subacute, fleshy, upper surface basally grooved. Column c. $1.2 \mathrm{~mm}$ long; columnfoot c. $1.5 \mathrm{~mm}$ long. Pedicel and ovary $5-7 \mathrm{~mm}$ long.

Distribution: Nepal, North-eastern India, Bhutan, northern Myanmar (Kachin State), China (Yunnan, Guangxi, Hunan, Guangdong, Jiangxi, Fujian, Zhejiang), Thailand, Laos, Vietnam.

Specimens examined: MYANMAR, Kachin State: Nogmung Township, 12.2015, Saw Lwin KSL1082 (RAF); North-eastern part, $1065 \mathrm{~m}, 2.1912$, Toppin 6265 (K); Chipwi Township, 2135 m, 5.1925, Forrest 26603 (K, E).

Notes: Seidenfaden (1973) identified a specimen of the synonymous Bulbophyllum insulsum (Gagnep.) Seidenf. from Upper Myanmar (Forrest 26603, $\mathrm{K})$ as possibly belonging to this species which is confirmed here.

Bulbophyllum striatum (Griff.) Rchb.f. in W.G. Walpers, Ann. Bot. Syst. 6: 257. 1861. Dendrobium striatum Griff., Not. Pl. Asiat. 3: 318. 1851. Sarcopodium striatum (Griff.) Lindl., Fol. Orchid. 2: 5. 1853. Phyllorkis striata (Griff.) Kuntze, Revis. Gen. Pl. 2: 678. 1891.

Type: INDIA, Assam, Mumbree, 11.11.1835, Griffith 236/146 (Holotype, K-LINDL!).
Epiphytic herbs, pseudobulbs spaced at intervals of $1.5-10 \mathrm{~cm}$ on a creeping rhizome, ovoid or globose, $0.8-1.7 \times 0.7-1.7 \mathrm{~cm}$, one-leaved. Leaves oblong, prominently petiolate, $2.3-8 \times 1.2-3.8$ $\mathrm{cm}$, obtuse. Inflorescences erect, $2.5-15 \mathrm{~cm}$ long; peduncle slender, $1.7-13 \mathrm{~cm}$ long; racemes usually subumbellate with 2 to 4 flowers; floral bracts lanceolate, 4-7 mm long, acute. Flowers greenish or yellowish green with purple veins. Median sepal elliptic-ovate, 9-12 × 3.3-4.5 mm, acute; lateral sepals free, triangular, $8.5-13.5 \times 3.5-5 \mathrm{~mm}$. Petals narrowly elliptic-ovate, $4.5-7 \times 1.8-2.4 \mathrm{~mm}$, acute. Lip ovate-elliptic, 5-8 $\times 2.2-4 \mathrm{~mm}$, obtuse, minutely auriculate at base. Column $2.8-3.5 \mathrm{~mm}$ long; stelidia rounded, short. Pedicel and ovary 10-17 mm long.

Distribution: Nepal, north-eastern India, Bhutan, northern Myanmar (Kachin State), Thailand, northern Vietnam, South-western China (Yunnan).

Specimens examined: MYANMAR, Kachin State: Nogmung Township, 12.2015, Saw Lwin KSL 1063 (RAF).

Bulleyia yunnanensis Schltr., Notes Roy. Bot. Gard. Edinburgh 5: 108. 1912.

Type: CHINA, Yunnan, Tali Range, 2440-2745 m, 7.8.1906, Forrest 4879 (Holotype, E!; Isotype, BM).

Epiphytic herbs, pseudobulbs adjacent, narrowly ovoid, 3.5-7 × 1-2 cm, two-leaved. Leaves lanceolate, petiolate, $16-43 \times 1.5-3 \mathrm{~cm}$, longacuminate. Inflorescences arching; peduncle slender, 30-66 cm long; racemes 6-16 cm long, with 10 to 15 laxly arranged flowers; floral bracts ovate, $15-20 \times 12-15 \mathrm{~mm}$, acute. Flowers white with light brown lip. Median sepal ovate-oblong, 12-14 × 4-5 mm, acute; lateral sepals similar to the median sepal. Petals elliptic-oblong, 15-18 $\times 2-3 \mathrm{~mm}$, acute. Lip entire, $12-14 \times 5-6 \mathrm{~mm}$, spurred; hypochile elliptic-ovate, concave; epichile reniform, emarginate; disk keeled. Column 8-10 $\mathrm{mm}$ long. Pedicel and ovary 6-7 $\mathrm{mm}$ long.

Distribution: North-eastern India, Bhutan, northern Myanmar (Kachin State), South-western China (Yunnan).

Specimens examined: MYANMAR, Kachin State: Chipwi Township, 30.7.1914, Kingdon-Ward 1837 (E), det. E.F. de Vogel.

Notes: The specimen cited above was identified as belonging to this species already in February 1988, but was apparently overlooked by later students working on the orchids of Myanmar. 
Calanthe alpina Hook.f. ex Lindl., Fol. Orchid. 6: 4. 1854. Alismorkis alpina (Hook.f. ex Lindl.) Kuntze, Revis. Gen. Pl. 2: 650. 1891.

Type: INDIA, Sikkim, Lachen, Hooker 245 (Holotype, K-LINDL; possible Isotype, AMES!, P!).

Terrestrial herbs, pseudobulbs insignificant and largely hidden by the leaf bases. Leaves 3, well developed during flowering, evergreen, elliptic, $11-26 \times 3-6(-9) \mathrm{cm}$, acute or obtuse. Inflorescences 1 or rarely 2, basal, erect, $30-50 \mathrm{~cm}$ long, puberulent; racemes $3-12 \mathrm{~cm}$ long, with 3 to more than 10 laxly or subdensely arranged flowers; floral bracts persistent, lanceolate, c. $15 \mathrm{~mm}$ long, glabrous, acuminate. Flowers yellow-green with purplish markings. Median sepal elliptic, 15-20 $\times$ 5-6 mm, aristate; lateral sepals lanceolate, 15-20 × 6-7 mm, aristate. Petals oblong to lanceolate, 12-13 $\times 4-4.5 \mathrm{~mm}$, acute. Lip basally united with column, suborbicular, to $10 \times 17 \mathrm{~mm}$, margin fimbriate; spur cylindric, 15-35 mm long. Column c. $8 \mathrm{~mm}$ long. Pedicel and ovary c. $20 \mathrm{~mm}$ long, puberulent.

Distribution: India (Himalayas, Nagaland), Nepal, Bhutan, Upper Myanmar, China (Xizang, Sichuan, Yunnan, Gansu, Shaanxi, Hubei), Taiwan, Japan (Honshu, Shikoku).

Specimens examined: MYANMAR, Upper Myanmar, 2440 m, 8.6.1914, Kingdon-Ward 1653 (E); Kachin State: Triangle, 2895 m, 3.7.1953, Kingdon-Ward 21170 (AMES).

Calanthe arcuata Rolfe, Bull. Misc. Inform. Kew 1896: 196. 1896.

Type: CHINA, Hubei, Hsingshan, Henry 6514 (Holotype, K!; Isotype, AMES!).

Terrestrial herbs, pseudobulbs insignificant and largely hidden by the leaf bases. Leaves 3 or 4 , well developed during flowering, evergreen, narrowly lanceolate, $15-28 \times 0.7-3 \mathrm{~cm}$, acute. Inflorescences 1 or 2, basal, erect, $30-50 \mathrm{~cm}$ long, puberulent; racemes $10-25 \mathrm{~cm}$ long, with 10 laxly arranged flowers; floral bracts persistent, lanceolate, 10-18 $\mathrm{mm}$ long, glabrous. Flowers yellow-green to white. Sepals lanceolate, 17-22 $\times$ c. $4 \mathrm{~mm}$, acuminate. Petals linear, $17-22 \times 2-3$ $\mathrm{mm}$, acuminate. Lip basally united with column, prominently or weakly 3-lobed, 11-18 $\times 4-6 \mathrm{~mm}$; side lobes oblong; mid-lobe rhombic, 8-10 × 6-7 $\mathrm{mm}$, aristate; disk with 3-5 keels; spur cylindric, $c$. $5 \mathrm{~mm}$, long. Column thick, 4-5 mm long, glabrous or puberulent. Pedicel and ovary 14-20 mm long, puberulent.
Distribution: Northern Myanmar (Kachin State), China (Xizang, Yunnan, Sichuan, Gansu, Shaanxi, Guizhou, Hubei, Hunan), Taiwan.

Specimens examined: MYANMAR, Kachin State, Chipwi Township, 2440 m, 7.1914, Kingdon-Ward 1654 (E); Ibid., Kingdon-Ward 1696 \& 1721 (E).

Calanthe simplex Seidenf., Dansk Bot. Ark. 29 (2): 42. 1975. Type: THAILAND, Chiang Mai Province, Doi Chiang Dao, Put 4470 (Holotype, K!).

Terrestrial herbs, pseudobulbs insignificant and largely hidden by the leaf bases. Leaves $2-3$, well developed during flowering, evergreen, lanceolateoblong, 20-60 × 3.2-10.5 cm, acute. Inflorescences basal, erect, 40-70(-94) cm long, stout, puberulent; peduncle sheathed; racemes $20-32 \mathrm{~cm}$ long, with up to 30 laxly arranged flowers; floral bracts persistent, lanceolate, $10-25 \mathrm{~mm}$ long, puberulent, acuminate. Flowers yellow-green; hirsute. Median sepal elliptic, 9-14 × 5-7 mm, acute; lateral sepals similar, oblique. Petals spathulate, obovateelliptic, 8-12 × 3.5-5.3 mm, acute. Lip basally united with column, broadly reniform, c. $10 \times 16$ $\mathrm{mm}$, slightly emarginate; disk basally with three ridges; spur cylindric, 8-14 mm long. Column 4-8 $\mathrm{mm}$ long, hirsute. Pedicel and ovary c. $15 \mathrm{~mm}$ long, puberulent.

Distribution: North-eastern India (Manipur), western Myanmar (Chin State), northern Thailand, South-western China (Yunnan).

Specimens examined: MYANMAR, Chin State, without locality, 1645 m, 9.10.1923, Daun 101 (K); Haka District, 2135 m, 13.12.1909, Venning 1 (K).

Notes: Seidenfaden (1975: 42-45) argued that this species is superficially similar to Calanthe wardii W.W. Sm. from Upper Myanmar (currently considered conspecific with C. whiteana King \& Pantl.), but decided to keep it separate because of densely hirsute sepal outsides and a stiff-hairy column (vs. sepal outsides only with fine hairs and column glabrous in C. wardii).

Chrysoglossum ornatum Blume, Bijdr. Fl. Ned. Ind.: 338. 1825.

Type: INDONESIA, Java, Mount Salak, Tjapus River, Blume s.n. (Holotype, L, not located; Isotype K [fide Pearce \& Cribb, 2002: 308]).

Terrestrial herbs, pseudobulbs spaced at intervals of 1-2 cm on a creeping rhizome, cylindric, conic, 5-7 × 8-20 cm, one-leaved. Leaves narrowly elliptic, plicate, prominently petiolate, 20-34 × $4.5-7.5 \mathrm{~cm}$, acuminate, papery. Inflorescences 
27-53(-75) cm long; peduncle slender, sheathed; racemes $4.5-19 \mathrm{~cm}$ long, with 10 or more laxly arranged flowers; floral bracts lanceolate, 10-14 $\mathrm{mm}$ long, acute. Flowers: sepals green with red dots, lip white or yellow. Median sepal lanceolate, $10.9-17.6 \times 3.3-4.8 \mathrm{~mm}$, subacute; lateral sepals falcately lanceolate, $9.3-15.2 \times 3-5 \mathrm{~mm}$, subacute. Petals lanceolate, 9.7-15.4 $\times 4.8-6.0 \mathrm{~mm}$, subacute. Lip 3-lobed, 8.5-11 × 6.8-10.4 mm; side lobes erect, 2.1-4.3 mm wide, obtuse; mid-lobe ovate, concave, 4-7.9 mm wide, subacute; disk with 3 keels. Column 6-8 mm long, winged; with two auricles near middle; column-foot $1.5-2.3 \mathrm{~mm}$ long, with conical spur $0.5-1.6 \mathrm{~mm}$ long. Pedicel and ovary $c$. $20 \mathrm{~mm}$ long.

Distribution: Sri Lanka, India (eastern coast), Nepal, Bhutan, northern Myanmar (Kachin State), Thailand, Laos, Cambodia, Vietnam, southwestern and southern China (Yunnan, Guangxi, Hainan), Taiwan, Malesia, Pacific islands.

Specimens examined: MYANMAR, Kachin State, Nogmung Township, 460 m, 23.4.1926, KingdonWard $6692(\mathrm{~K})$.

Notes: Already in a recent revision of the genus it was pointed out that, based on the general distribution of the species, it could possibly also occur in Myanmar (van der Burgh \& de Vogel, 1997: 143).

Cleisostoma duplicilobum (J.J. Sm.) Garay, Bot. Mus. Leafl. 23: 171. 1972. Sarcanthus duplicilobus J.J. Sm., Bull. Dép. Agric. Indes Néerl. 13: 64. 1907. Garayanthus duplicilobus (J.J. Sm.) Szlach., Fragm. Florist. Geobot., Suppl. 3: 136. 1995.

Type: INDONESIA, Java, Dieng Plateau, Kamerling s.n. (Holotype, BO [fide O'Byrne, 2012: 69]).

Monopodial epiphytic herbs with elongate stems, many-leaved. Leaves strap-shaped, 13-18 $\times 1.5-2.6 \mathrm{~cm}$, obtuse, apically unequally bilobed. Inflorescences horizontal or pendulous, branched; peduncle slender, $c .10 \mathrm{~cm}$ long; all branches with many laxly arranged flowers. Flowers pale yellow with reddish brown blotches and stripes, lip white. Median sepal ovate-lanceolate, c. $5 \times 3 \mathrm{~mm}$, subacute; lateral sepals oblong, c. $4.7 \times 3.3 \mathrm{~mm}$, obtuse. Petals narrowly oblong-ovate, c. $4 \times 1.3$ $\mathrm{mm}$, obtuse. Lip 3-lobed, spurred; side lobes ovate, erect, curving inwards, acute; mid-lobe triangular, basally auriculate; spur with longitudinal septum and large hairy backwall callus, c. $5 \mathrm{~mm}$ long and $3.3 \mathrm{~mm}$ broad. Column c. $2 \mathrm{~mm}$ long.

Distribution: North-eastern India (Nagaland), western Myanmar (Chin State), Thailand, Laos, Vietnam, Malesia.
Specimens examined: MYANMAR, Chin State, Falam District, 1525 m, 9.10.1923, Daun 74 (K).

Crepidium khasianum (Hook.f.) Szlach., Fragm. Florist. Geobot., Suppl. 3: 127. 1995. Microstylis khasiana Hook.f., Fl. Brit. India 5: 686. 1888. Malaxis khasiana (Hook.f.) Kuntze, Revis. Gen. Pl. 2: 673. 1891.

Type: INDIA, Khasia Hills, 1220-1525 m, Lobb s.n. (Holotype, K! [barcode K000387678]).

Terrestrial herbs, stems fleshy, mostly 4- or 5-leaved. Leaves ovate-lanceolate, shortly petiolate, $3.5-7 \times$ $1.5-2 \mathrm{~cm}$, acuminate. Inflorescences erect; peduncle slender, 8-11 cm long; racemes 4-5 cm long, with 20 or more laxly arranged flowers; floral bracts lanceolate, 2.5-3 mm long, acute. Flowers greenish yellow; not resupinate. Sepals elliptic, 2.4-2.8 $\times$ 1-1.2 mm, obtuse; lateral sepals oblique. Petals linear, 2-2.5 × c. $0.3 \mathrm{~mm}$, obtuse. Lip oblong, 3.5-4 $\mathrm{mm}$ long, both sides indented near the middle, base with a hollow depression, apically slightly bilobed; auricles oblong, stretching backwards behind column. Column c. $1 \mathrm{~mm}$ long. Pedicel and ovary 3.5-4.5 mm long.

Distribution: Nepal, north-eastern India, western Myanmar (Chin State), northern Thailand, Southwestern China (Yunnan).

Specimens examined (det. S. Watthana): MYANMAR, Chin State, southern Chin Hills, 1550 m, 2.8.2008, Fujikawa, Kano, Matsumoto, Yasuda, Ling Shein Man \& Hong Mang 053114 (MBK); Ibid., 1400 m, 25.7.2008, Yasuda 053605 (MBK); Ibid., 9.8.2008, Yasuda 060072 (MBK); Ibid., 1400-1600 m, 26.6.2009, Tanaka $\mathcal{E}$ Yukawa 081194 (MBK); Ibid., 1430 m, 24.6.2011, Ling Shein Man, Ha Shein Aung \& Htun Tin 087417 (MBK); Ibid., 1660 m, 12.7.2013, Mu Mu Aung, Law Shine \& Law Shein Aung 092458 (MBK); Ibid., 1370 m, 8.7.2013, Ling Shein Mang 093057 (MBK); Ibid., 1590-1965 m, 22.8.2013, Fujikawa, Srisanga, Maknoi, Ling Shein Man \& Law Shine 094196 (MBK).

Crepidium szemaoense (Tang \& F.T. Wang) Nuammee, Seelanan, Suddee \& H.A. Pedersen, Thai Forest Bull., Bot. 44: 36. 2016. Malaxis szemaoensis Tang \& F.T. Wang, Acta Phytotax. Sin. 1: 75.1951.

Type: CHINA, Yunnan, Szemao, north-western mountains, 1525 m, 1901, Henry 13128 (Holotype, $\mathrm{K} !)$.

Terrestrial herbs, stems fleshy, 2-4-leaved. Leaves elliptic or ovate, petiolate, $2-6.5 \times 1.2-3.5 \mathrm{~cm}$, acute. Inflorescences erect; peduncle slender, 8-12 cm long; racemes $2.5-6 \mathrm{~cm}$ long, with 7 or 
8 laxly arranged flowers; floral bracts reflexed, lanceolate, 3-4 mm long. Flowers greenish yellow; not resupinate. Sepals oblong, 3-3.5 × c. $1 \mathrm{~mm}$, obtuse; lateral sepals similar to median one but oblique and slightly shorter. Petals linear, 3-3.5 $\times$ c. $0.4 \mathrm{~mm}$, obtuse. Lip broadly oblong, c. $5 \times 3.5$ $\mathrm{mm}$, base with a hollow depression, apically with several irregular teeth; auricles oblong, stretching backwards behind column. Column c. $1 \mathrm{~mm}$ long. Pedicel and ovary 3-4 mm long.

Distribution: Western Myanmar (Chin State), northern Thailand, south-western China (Yunnan).

Specimens examined: MYANMAR, Chin State, southern Chin Hills, $1685 \mathrm{~m}$, 8.7.2013, Mu Mu Aung, Law Shine, Law Shein Aung \& Khin Win Maung 092359 (MBK).

Notes: Because of similar floral features previously regarded as conspecific with the Sumatran Crepidium ovalisepalum (J.J. Sm.) Szlachetko (Seidenfaden, 1978; Chen et al., 2009). The confusion was cleared up by Nuammee et al. (2016).

Dendrobium numaldeorii C. Deori, Hynn. \& Phukan, Orchid Rev. 112 (1259, Suppl.): 279. 2004.

Type: INDIA, Arunachal Pradesh, Dibang Valley District, Mehao Wildlife Sanctuary, $1300 \mathrm{~m}$, 10.10.2003, Deori 101,122 (Holotype, CAL; Isotype, ASSAM).

Epiphytic herbs, stems $2.5-10 \mathrm{~cm}$ long, leafy in the upper part. Leaves distichous, narrowly oblong, 2-6.6 $\times 0.5-1.3 \mathrm{~cm}$, obtuse. Inflorescences $4.5-13 \mathrm{~cm}$ long, borne axillary and at the stem apex, pendent, longer than the leaves; each raceme with 4 to 16 laxly arranged flowers; floral bracts lanceolate, 3-4.5 × 1-1.5 mm, acuminate. Flowers greenish or brownish yellow with maroon tinge. Sepals very narrowly lanceolate and caudate-acuminate; median sepal $18-20 \times 2-2.5 \mathrm{~mm}$; lateral sepals 20-23 × 8-9 mm. Petals needle-like, 13-16 × 1.5-2 $\mathrm{mm}$. Lip shallowly 3-lobed, shorter than sepals, 13-14 × 6-6.5 mm; side lobes rounded, margins lacerate; mid-lobe triangular, acute; disk with three fleshy ridges. Column 4-5 mm long; columnfoot c. $10 \mathrm{~mm}$ long. Pedicel and ovary $8-9 \mathrm{~mm}$ long.

Distribution: North-eastern India (Arunachal Pradesh), Northern Myanmar (Kachin State).

Specimens examined: MYANMAR, Kachin State, Chipwi Township, 1370 m, 13.12.1938, KingdonWard 108 (AMES, NY).

Galearis wardii (W.W. Sm.) P.F. Hunt, Kew Bull. 26: 173. 1971. Orchis wardii W.W. Sm., Notes Roy. Bot. Gard. Edinburgh 13: 215. 1921.
Types: CHINA, Yunnan, Atuntzu and Doker-la at the Tibetan frontier, 3350 m, 6.1913, Kingdon-Ward 447 (Syntype, E!); Ibid., 6.1913, Kingdon-Ward 589 (Syntype, E!).

Terrestrial herbs with creeping underground rhizome, two-leaved. Leaves 7-15 × 2.5-4.5 cm, obtuse, sometimes mucronate. Inflorescences erect, $3.5-8 \mathrm{~cm}$ long, stout; racemes $c .3 \mathrm{~cm}$ long, with 5-10 or more semi-densely arranged flowers; floral bracts lanceolate, longer than the flowers, c. $35 \mathrm{~mm}$ long, acuminate. Flowers spreading, whitish pink to purple with dark purple spots. Sepals similar, ovate-lanceolate, 8-9 × 3-3.5 mm, obtuse; median sepal erect; lateral sepals spreading or reflexed. Petals erect and forming a hood with the median sepal, ovate-lanceolate, c. $7 \times 2.5 \mathrm{~mm}$, acute. Lip ovate or suborbicular, spurred, $8-9 \times 8-9 \mathrm{~mm}$, rounded-obtuse; spur cylindric, 7-10 mm long. Pedicel and ovary 10-12 mm long.

Distribution: Northern Myanmar (Kachin State), south-western China (Xizang, Yunnan, Sichuan).

Specimens examined: MYANMAR, Kachin State, Nogmung Township, 3660 m, 28.6.1926, KingdonWard 7007 (K); Ibid., 22.6.1931, Kingdon-Ward 9678 (BM).

Goodyera hemsleyana King \& Pantl., J. Asiat. Soc. Bengal, Pt. 2, Nat. Hist. 64: 342. 1895. Epipactis hemsleyana (King \& Pantl.) A.A. Eaton, Proc. Biol. Soc. Washington 21: 64. 1908.

Lectotype (designated by Bhattacharjee \& Bhakat, 2010: 73): INDIA, West Bengal, Darjeeling, Senchal, $2135 \mathrm{~m}, 7.1892$, Pantling 215 (CAL; Isolectotype, AMES!, CAL, BM, K!, P, W) [fide Bhattacharjee \& Chowdhery, 2012: 503].

Terrestrial herbs with creeping rhizome, 3-5-leaved. Leaves ovate, petiolate, 2-4.5 × 1-2 $\mathrm{cm}$, acute, dark green with white reticulation. Inflorescences erect, terminal; racemes $2-4 \mathrm{~cm}$ long, with 2 to 7 laxly arranged flowers; floral bracts lanceolate, 10-15 mm long, longer than the flowers, acuminate, glandular-pubescent. Flowers white with reddish-brown tinge; secund. Sepals subsimilar, 6-9 mm long; median sepal oblonglanceolate, pubescent; lateral sepals ovate. Petals oblong-lanceolate, 6-7 mm long. Lip oblong, 7-8 $\mathrm{mm}$ long, base saccate, apex obtuse. Column 2-3 $\mathrm{mm}$ long. Pedicel and ovary $5-9 \mathrm{~mm}$ long.

Distribution: Nepal, north-eastern India (Darjeeling), Bhutan, Northern Myanmar (Kachin State), South-western China (Yunnan). 
Specimens examined: MYANMAR, Kachin State, Chipwi Township, 1830 m, 24.8.1914, KingdonWard 1897 p.p. (E [barcode 00743118]), det. Huaizhen Tian.

Habenaria intermedia D. Don, Prodr. Fl. Nepal.: 24. 1825. Kryptostoma intermedium (D. Don) Olszewski \& Szlach., Ann. Bot. Fenn. 37: 299. 2000. Ochyrorchis intermedia (D. Don) Szlach., Richardiana 4: 55. 2004.

Possible type: NEPAL, Gosainthan, Wallich's Catalogue 7030 (K-W!).

Terrestrial herbs with ellipsoid underground root tubers, stems stout, erect. Leaves $3-5$, ovatelanceolate, 3.5-8 $\times 2-4 \mathrm{~cm}$, acute, with amplexicaul base. Inflorescences terminal, erect; racemes 6-15 cm long, with 1 to 4 laxly arranged flowers; floral bracts ovate, $4-5 \mathrm{~cm}$ long, about as long as the ovary, acuminate. Flowers white or greenish white. Sepals with ciliate margins; median sepal erect, ovate-oblong, to $25 \times 12 \mathrm{~mm}$, acute; lateral sepals similar to the median sepal but longer and narrower, obliquely falcate-lanceolate. Petals erect and forming a hood with the median sepal, obliquely subovate-falcate, c. $22 \times 8 \mathrm{~mm}$, acute. Lip deeply 3-lobed, 28-30 mm long, spurred, lobes ciliate; side lobes linear, outer margin with up to 10 filiform lobules; mid-lobe linear, 18-20 mm long, acute; spur cylindric, 70-85 mm long, exceeding ovary. Column c. $7 \mathrm{~mm}$ long; connective c. $2 \mathrm{~mm}$ wide; stigmas elongate, clavate. Pedicel and ovary 38-45 mm long.

Distribution: Pakistan, India (Himalayas from Kashmir to North-eastern India), Nepal, western Myanmar (Chin State), South-western China (Xizang).

Specimens examined (all collections except the first one det. S. Watthana): MYANMAR, Chin State, southern Chin Hills, 1830-1980 m, 21.6.1956, U Mg Gale-2 \& U Chit Ko Ko 5595 (RAF); Ibid., 2345 m, 9.8.2008, Yasuda 060067 (MBK), specimen in fruit and identification after a photograph; Ibid., 2500 $\mathrm{m}, 24.6 .2009$, Tanaka \& Yukawa 081134 (MBK); Ibid., 2030-2295 m, 27.6.2009, Tanaka \& Yukawa 081279 (MBK); Ibid., $2500 \mathrm{~m}, 28.6 .2009$, Tanaka E Yukawa 081398 (MBK); Ibid., 2500 m, 5.7.2013, Mu Mu Aung, Law Shine E Aung Htay 092340 (MBK).

Habenaria linguella Lindl., Gen. Sp. Orchid. Pl.: 325. 1835. Habenaria acuifera var. linguella (Lindl.) Finet, Rev. Gén. Bot. 13: 525. 1901.

Lectotype (designated by Seidenfaden, 1977): CHINA, Kwangtung, Millet s.n. (K).
Terrestrial herbs with underground root tubers, stems stout, erect. Leaves 3-4, narrowly oblong or oblong-lanceolate, $5-12(-27) \times 1.2-2 \mathrm{~cm}$, with amplexicaul base, acuminate. Inflorescences terminal, erect; racemes $2.5-6 \mathrm{~cm}$ long, with 9 to 20 densely arranged flowers; floral bracts linearlanceolate, 12-25 mm long. Flowers yellow, often with brownish tinge. Median sepal erect, forming a hood with the petals, broadly elliptic, $4-5 \times$ 3-3.5 mm, obtuse; lateral sepals reflexed, obovate, 6-7 $\times 4-4.5 \mathrm{~mm}$, subacute or obtuse. Petals erect, obliquely ovate or elliptic, $4-5 \times 2-2.5 \mathrm{~mm}$, obtuse. Lip deeply 3-lobed, 9-10 mm long, spurred, with a collar around the spur mouth; side lobes subulate, 1.5-2.8 mm long, acuminate; mid-lobe linear, 8-9 mm long, obtuse; spur cylindric, 20-29 mm long, exceeding ovary. Column c. $2 \mathrm{~mm}$ long; connective narrow; stigmas oblong, c. $1.5 \mathrm{~mm}$ long. Pedicel and ovary $18-23 \mathrm{~mm}$ long.

Distribution: Eastern Myanmar (Shan State), Vietnam, South-western and southern China (Yunnan, Guizhou, Guangxi, Guangdong, Hainan, Hong Kong).

Specimens examined: MYANMAR, Shan State: Loilem District, $1220 \mathrm{~m}$ and above, 1910, Robertson $7(\mathrm{~K})$.

Habenaria pantlingiana Kraenzl., Orchid. Gen. Sp. 1: 892. 1901.

Type: INDIA, Sikkim, Pantling 415 (Holotype, K!; Isotype, BM, W [fide Pearce \& Cribb, 2002: 151]).

Terrestrial herbs with oblong underground root tubers, stems stout, erect, with 5-7 leaves clustered near the stem middle. Leaves ovate-elliptic, (7.5-)9$15 \times 2.5-4.8 \mathrm{~cm}$, acute to acuminate. Inflorescences terminal, erect, with sterile bracts; racemes 8-20 cm long, 20-30-flowered; floral bracts ovatelanceolate, $18-24 \times 2.5-4 \mathrm{~mm}$, about as long as the ovary, long-acuminate. Flowers green, lip and petals sometimes whitish; sepals and petals with long filiform caudae. Median sepal erect, ovatelanceolate, $15-30 \times 4-6 \mathrm{~mm}$; lateral sepals spreading or reflexed, ovate-lanceolate, $15-29 \times 3.5-6 \mathrm{~mm}$. Petals deeply 2-lobed, each lobe filiform; posterior lobe 11-17 mm long; anterior lobe 8-20 mm long. Lip deeply 3-lobed, lobes filiform, 14-28 × c. 0.8 $\mathrm{mm}$; spur cylindric, 19-23 $\mathrm{mm}$ long, exceeding ovary. Column 1.8-2 mm long; stigmas clavate. Pedicel and ovary 18-22 mm long.

Distribution: Nepal, North-eastern India (Sikkim, Arunachal Pradesh, Nagaland), eastern Myanmar (Shan State), northern Thailand, Vietnam, southern China (Guangxi, Guangdong, Hainan), Taiwan, southern Japan (Nansei-shoto). 
Specimens examined: MYANMAR, Shan State, south-western part, 1200 m, 3.9.2014, Seo, Miyake, Aung Zaw Moe, Aya Mya Mon \& Thin Su Tin 100743 (MBK).

Herminium fallax (Lindl.) Hook.f., Fl. Brit. India 6: 129. 1890. Peristylus fallax Lindl., Gen. Sp. Orchid. Pl.: 298. 1835. Habenaria fallax (Lindl.) King \& Pantl., Ann. Roy. Bot. Gard. (Calcutta) 8: 325. 1898. Platanthera fallax (Lindl.) Schltr., Repert. Spec. Nov. Regni Veg. Beih. 4: 111. 1919. Monorchis fallax (Lindl.) O. Schwarz, Mitt. Thüring. Bot. Ges. 1: 95.1949.

Type: NEPAL, 1821, Wallich's Catalogue 7412 (Holotype, K-LINDL; Isotype, K-W!) [fide Raskoti et al., 2017: 35].

Terrestrial herbs with subglobose underground root tubers, stems slender or somewhat stout, erect, one-leaved. Leaves oblong-lanceolate, $6-13 \times 0.8-2$ $\mathrm{cm}$, acute. Inflorescences terminal, erect, 11-22 cm long; racemes 5-14 cm long, densely manyflowered; floral bracts lanceolate, 6-15 mm long, acuminate. Flowers yellowish green. Median sepal suberect, oblong-ovate, $2-4 \times 1.5-2.1 \mathrm{~mm}$, obtuse; lateral sepals obliquely ovate, $3-4.2 \times 1-1.5 \mathrm{~mm}$, subacute. Petals ovate-lanceolate, $2.5-5 \times 1-1.5$ $\mathrm{mm}$, acute. Lip 3-lobed, 3-5.5 × 1-2 mm, spurred, ecallose; side lobes oblong, c. $1.5 \times 0.5 \mathrm{~mm}$, obtuse; mid-lobe oblong, $1-2 \times$ c. $2 \mathrm{~mm}$, obtuse; spur oblong-clavate, $0.7-1.5 \mathrm{~mm}$ long. Column 1.5-2 $\mathrm{mm}$ long. Pedicel and ovary 6-7 $\mathrm{mm}$ long.

Distribution: India (North-western Himalayas, Sikkim), Nepal, Bhutan, western Myanmar (Chin State), South-western China (Xizang, Yunnan, Sichuan).

Specimens examined (det. H. Kurzweil \& B.B. Raskoti): MYANMAR, Chin State, southern Chin Hills, 2000 m, 5.8.2008, Yasuda 053610A (MBK); Ibid., 7.8.2008, Yasuda 060088 (spirit collection MBK); Ibid., 1900 m, 11.8.2008, Yasuda 053613 (MBK, spirit collection MBK); Ibid., $3070 \mathrm{~m}$, 30.7.2013, Mu Mu Aung, Ling Shein Mang, Aung Htay \& Har Shin Aung 092824 (MBK); Ibid., 2400 m, 21.8.2013, Fujikawa, Srisanga, Maknoi, Ling Shein Man \& Law Shine 094119 (MBK); Ibid., 10.8.2016, Kurzweil s.n., photographic record.

Notes: The collections Yasuda 053610A, 053613 \& 060088 and Fujikawa et al. 094119 differ from typical Herminium fallax in having wider leaves and many-flowered slender inflorescences with flexible axes, but have been interpreted as merely variations of $H$. fallax (B.B. Raskoti, pers. comm.).
Herminium forceps (Finet) Schltr., Notes Roy. Bot. Gard. Edinburgh 5: 97.1912. Peristylus forceps Finet, Rev. Gén. Bot. 13: 521. 1901. Habenaria forceps (Finet) Schltr., Repert. Spec. Nov. Regni Veg. Beih. 4: 127. 1919.

Lectotype (designated by Raskoti et al., 2017: 37): CHINA, Yunnan, in the mountains, 7.1897, Ducloux 319 (P!; Isolectotype, P!).

Terrestrial herbs with ovoid-oblong underground root tubers; stems slender, erect, 2-5-leaved. Leaves widely spaced, oblong-lanceolate, 4-10 × $0.5-2.3 \mathrm{~cm}$, acute. Inflorescences terminal, erect, 10-30 cm long; racemes 3-20 cm long, densely many-flowered; floral bracts lanceolate, 5-20 $\mathrm{mm}$ long, longer than ovary, acuminate. Flowers green. Median sepal erect, oblong-ovate, 2-3.5 × 1.5-3 mm, obtuse; lateral sepals oblong-lanceolate, oblique, $2-3.5 \times 1-1.5 \mathrm{~mm}$, obtuse. Petals ovatelanceolate, oblique, 2-4 × 1-1.6 mm, obtuse. Lip ligulate, 3-5 × 1-1.5 mm, obtuse, spurred, ecallose; spur pendulous, $0.8-1 \mathrm{~mm}$ long, shorter than the ovary. Column c. $1.5 \mathrm{~mm}$ long; stigma oblong, situated below the rostellum. Pedicel and ovary 4-10 mm long, shortly beaked.

Distribution: Eastern Myanmar (Shan State), Southwestern and central China (Xizang, Yunnan, Sichuan, Gansu, Guizhou, Hubei).

Specimens examined: MYANMAR, Shan State, south-western part, 1530 m, 27.9.2015, Kilgour, Kertsawang, Ruchisansakun \& Phyu Phyu Hnin m-760 (MBK).

Herminium kamengense A.N. Rao, J. Econ. Tax. Bot. 25 (2): 287. 2001.

Type: INDIA, Arunachal Pradesh, West Kameng District, Bomdila, 2500 m, Rao 30600A (Holotype, Orchid Herbarium Tipi).

Terrestrial herbs with underground root tubers, stems slender, erect, 2-3-leaved. Leaves narrowly lanceolate, c. $27 \times 1.5 \mathrm{~cm}$ though often much shorter, acuminate. Inflorescences terminal, erect, c. $24 \mathrm{~cm}$ long; racemes about $6-12 \mathrm{~cm}$ long, manyflowered; floral bracts ovate-lanceolate, 6-10 × 1.5$2 \mathrm{~mm}$, acuminate, longer than the ovary. Flowers green. Median sepal erect, ovate, 1.5-2.5 × 1-1.5 $\mathrm{mm}$, obtuse; lateral sepals obliquely ovate, $2-3 \times$ 1.4-2 mm, obtuse. Petals oblong-lanceolate, $2-3 \times$ $0.7-1 \mathrm{~mm}$, subacute. Lip broadly ovate, 3-lobed, c. $4 \times 3 \mathrm{~mm}$; side lobes oblong, c. $1.5 \times 0.5 \mathrm{~mm}$; midlobe triangular, c. $0.5 \times 0.5 \mathrm{~mm}$ long. Column about $1 \mathrm{~mm}$ long; stigma oblong, situated below the rostellum. Pedicel and ovary 6-9 $\mathrm{mm}$ long. 
Distribution: Nepal, North-eastern India (Arunachal Pradesh), western Myanmar (Chin State), China.

Specimens examined: MYANMAR, Chin State: southern Chin Hills, 10.8.2016, Kurzweil s.n., photographic record, det. B.B. Raskoti \& H. Kurzweil.

Herminium souliei (Finet) Rolfe, J. Linn. Soc., Bot. 36: 51. 1903. Herminium angustifolium var. souliei Finet, Rev. Gén. Bot. 13: 518. 1901. Monorchis souliei (Finet) O. Schwarz, Mitt. Thüring. Bot. Ges. 1: 96. 1949.

Type: CHINA, Sichuan, Tongolo, 18.8.1893, Soulié 407 (Holotype, P!; Isotype, K!).

Terrestrial herbs with oblong underground root tubers; stems erect, 2-4-leaved. Leaves widely spaced, lanceolate, $4-20 \times 0.5-2 \mathrm{~cm}$, acute. Inflorescences terminal, erect, $5-25 \mathrm{~cm}$ long; racemes $2.5-15 \mathrm{~cm}$ long, densely many-flowered; floral bracts ovate-lanceolate, 2-5 $\mathrm{mm}$ long, acuminate. Flowers pale green. Median sepal suberect, ovate, $2-3 \times 1.5-2 \mathrm{~mm}$, obtuse; lateral sepals obliquely ovate, $2.9-3.5 \times 1.2-2.5 \mathrm{~mm}$, subacute or obtuse. Petals erect, linear, $2-3 \times 0.4-$ $0.6 \mathrm{~mm}$, subacute or obtuse. Lip 3-lobed, 2.6-4.5 $\times$ 0.7-1.2 mm; side lobes linear-falcate, incurved, 1-2 $\mathrm{mm}$ long, obtuse; mid-lobe oblong-lanceolate, $c$. $0.5 \mathrm{~mm}$ long, obtuse. Column c. $1 \mathrm{~mm}$ long; stigma oblong, situated below the rostellum. Pedicel and ovary 5-10 mm long, shortly beaked.

Distribution: Nepal, eastern Myanmar (Shan State), South-western China (Xizang, Yunnan, Sichuan).

Specimens examined: MYANMAR, Shan State, south-western part, $1200 \mathrm{~m}$, 3.9.2014, Seo, Miyake, Aung Zaw Moe, Aya Mya Mon E Thin Su Tin 100687 (MBK).

Liparis mannii Rchb.f., Flora 55: 275. 1872. Leptorkis mannii (Rchb.f.) Kuntze, Revis. Gen. Pl. 2: 671. 1891. Stichorkis mannii (Rchb.f.) Marg., Szlach. \& Kulak, Acta Soc. Bot. Poloniae 77: 38. 2008.

Lectotype (designated by Seidenfaden, 1976): INDIA, Upper Assam, Mann 27 (W).

Epiphytic herbs, pseudobulbs adjacent, narrowly ovoid to suboblong, $1-2 \times 0.4-0.7 \mathrm{~cm}$, one-leaved. Leaves narrowly oblong-oblanceolate, indistinctly petiolate, $7-25 \times 0.6-1.2 \mathrm{~cm}$, acuminate, papery. Inflorescences terminal, erect, $9-13 \mathrm{~cm}$ long; peduncle slender; racemes $3.5-7.5 \mathrm{~cm}$ long, with 10 to many laxly arranged flowers; floral bracts lanceolate, 1.5-2.5 mm long. Flowers yellowish green. Sepals narrowly elliptic, $1.5-2 \times c .0 .7 \mathrm{~mm}$.
Petals linear, $1.3-1.8 \times$ c. $0.2 \mathrm{~mm}$. Lip ovate, deeply 3-lobed, to $2 \times 1.6 \mathrm{~mm}$; side lobes oblong-ovate, obtuse; mid-lobe rhombic, $0.6-0.8 \times$ c. $1 \mathrm{~mm}$, ecallose, irregularly denticulate, subacute. Column c. $1.2 \mathrm{~mm}$ long. Pedicel and ovary 1.5-2 mm long.

Distribution: North-eastern India (Sikkim, West Bengal, Assam), northern Myanmar (Kachin State), Laos, Vietnam, South-western China (Yunnan).

Specimens examined: MYANMAR, Kachin State, Tanai Township, 190-270 m, 6.12.2005, Murata, Kobayashi, Hayami \& Takashima 041223 (MBK).

Liparis petiolata (D. Don) P.F. Hunt \& Summerh., Kew Bull. 20: 52. 1966. Acianthus petiolatus D. Don, Prodr. Fl. Nepal.: 29. 1825.

Type: NEPAL, Wallich 1945 (Holotype, BM!) [fide Cribb \& Pearce, 2002: 202].

Terrestrial herbs, pseudobulbs spaced at intervals of $2-4 \mathrm{~cm}$ on a creeping rhizome, ovoid, 1.5-3 $\times 1-1.5 \mathrm{~cm}$, two-leaved. Leaves broadly ovate, $5-11 \times 3.5-8 \mathrm{~cm}$, with elongate petiole $5-11 \mathrm{~cm}$ long, acuminate, membranous or herbaceous. Inflorescences terminal, erect, $10-24 \mathrm{~cm}$ long; peduncle slender; racemes with up to 10 or more laxly arranged flowers; floral bracts lanceolate, 5-6 $\mathrm{mm}$ long, shorter than pedicel and ovary. Flowers greenish white, lip with purplish tinge. Sepals narrowly lanceolate, c. $8 \times 1.8 \mathrm{~mm}$. Petals linear, $7-8 \times$ c. $0.4 \mathrm{~mm}$. Lip ovate to elliptic, c. $10 \times 8-9 \mathrm{~mm}$, acute, mucronate, base with two prominent calli, margin sometimes irregularly incised. Column c. 2 $\mathrm{mm}$ long. Pedicel and ovary 6-8 $\mathrm{mm}$ long.

Distribution: Nepal, North-eastern India (Sikkim, Darjeeling, Khasia Mountains, Nagaland), Bhutan, northern Myanmar (Kachin State), northern Thailand, Vietnam, South-western and southern China (Xizang, Yunnan, Guangxi, Hunan, Jiangxi), Taiwan.

Specimens examined: MYANMAR, Kachin State, Chipwi Township, 2135-2440 m, 30.7.1914, Kingdon-Ward 1838 (E).

Liparis plantaginea Lindl., Gen. Sp. Orchid. Pl.: 29. 1830. Leptorkis plantaginea (Lindl.) Kuntze, Revis. Gen. Pl. 2: 671. 1891. Stichorkis plantaginea (Lindl.) Marg., Szlach. \& Kulak, Acta Soc. Bot. Poloniae 77: 39. 2008.

Type: INDIA, icon. Wallich 633 (Holotype, K) [fide Cribb \& Pearce, 2002].

Terrestrial herbs, pseudobulbs adjacent, narrowly ovoid, 2.5-6 × 1-1.5 cm, two-leaved. Leaves oblongelliptic to lanceolate, weakly petiolate, 19-22 × 
$3.5-4 \mathrm{~cm}$, acuminate, membranous or herbaceous. Inflorescences terminal, erect; peduncle slender; racemes $8-13 \mathrm{~cm}$ long, with up to 15 laxly arranged flowers; floral bracts lanceolate, 7-15 $\mathrm{mm}$ long, usually shorter than pedicel and ovary. Flowers green. Sepals similar, oblong-lanceolate, 8-10 $\times$ 2-4 mm, subacute. Petals linear, c. $10 \mathrm{~mm}$ long, obtuse. Lip suborbicular, shortly clawed, 8-10 × 7-9 $\mathrm{mm}$, base with two prominent calli, margin erose. Column c. $4 \mathrm{~mm}$ long, with small rounded apical wings. Pedicel and ovary $10-15 \mathrm{~mm}$ long.

Distribution: Central Nepal, North-eastern India (Sikkim, Darjeeling, Assam, Nagaland), Bhutan, northern Myanmar (Kachin State), Thailand (northern, North-eastern, peninsular), Vietnam.

Specimens examined: MYANMAR, Kachin State, Nogmung or Putao Township, 2007 or 2009, Kurzweil \& Saw Lwin KL s.n. (SING).

Liparis tschangii Schltr., Repert. Spec. Nov. Regni Veg. 19: 380. 1924.

Type: CHINA, Yunnan, Nanfautchoang (Yunpe), 7.1920, Tschang 32 (not known).

Terrestrial herbs, pseudobulbs ovoid, 1-2 × 0.7-1.3 $\mathrm{cm}$, two-leaved. Leaves broadly ovate to ovateelliptic, indistinctly petiolate, blade spreading horizontally, 5-13 $\times 2.5-7.3 \mathrm{~cm}$, acuminate, membranous or herbaceous. Inflorescences terminal, erect, 11-29 cm long, much exceeding leaves; peduncle slender, with sterile bracts; racemes with 10 to 20 laxly arranged flowers; floral bracts lanceolate, reflexed, 4-6 $\mathrm{mm}$ long, shorter than pedicel and ovary, acute. Flowers green. Median sepal narrowly lanceolate, $7-8 \times 2-3$ $\mathrm{mm}$, subacute; lateral sepals similar but somewhat shorter and broader, oblique. Petals linear, $7-8 \times c$. $0.5 \mathrm{~mm}$, obtuse. Lip ovate or broadly elliptic, $7-8 \times$ 5-7 mm, with a darker longitudinal ridge with two small basal calli, apex broadly rounded. Column 2.5-4 mm long, upper part winged. Pedicel and ovary 8-9 mm long.

Distribution: Myanmar (Bago Region), Thailand (northern, North-eastern, south-western), Laos, Vietnam, South-western China (Yunnan, Sichuan).

Specimens examined: MYANMAR, Bago Region: Pyay District, 100-600 m, 9.12.2006, Sugawara, OhiToma, Naiki \& Ito 036384 (MBK).

Notes: In Thailand better known under the heterotypic synonym Liparis sutepensis Rolfe ex Downie.
Malaxis cylindrostachya (Lindl.) Kuntze, Revis. Gen. Pl. 2: 673. 1891. Dienia cylindrostachya Lindl., Gen. Sp. Orchid. Pl.: 22. 1830. Microstylis cylindrostachya (Lindl.) Rchb.f. in W.G. Walpers, Ann. Bot. Syst. 6: 207. 1861.

Type: NEPAL, Sheopore, Wallich's Catalogue 1934 (Holotype, K-LINDL!; Isotype, K-W!).

Terrestrial herbs, pseudobulbs conic, c. $1 \times 1 \mathrm{~cm}$, one-leaved. Leaves elliptic or orbicular, $4-9 \times 3-4.5$ $\mathrm{cm}$, acute or obtuse, membranous or herbaceous. Inflorescences terminal, erect; peduncle slender, ridged, 5-10 cm long; racemes cylindric, (2.5-)5-14 cm long, with many densely arranged flowers; floral bracts lanceolate, 2-2.5 mm long, acute. Flowers yellowish green, not resupinate. Sepals similar, ovate, $2-3 \times 0.4-0.5 \mathrm{~mm}$, acute or acuminate. Petals linear-lanceolate, 1-1.9 × 0.4-0.5 $\mathrm{mm}$, acute. Lip broadly ovate, $1.5-2 \times 1-2 \mathrm{~mm}$, concave, fleshy, margin thickened, with a central ridge, margin denticulate, apex prominently mucronate. Column c. $0.5 \mathrm{~mm}$ long. Pedicel and ovary 1.5-2 $\mathrm{mm}$ long.

Distribution: Pakistan, India (North-western Himalayas, Sikkim), Nepal, Bhutan, western Myanmar (Chin State), Southern-western China (Xizang).

Specimens examined (all collections except the last one det. S. Watthana): MYANMAR, Chin State, southern Chin Hills, 2800 m, 13.8.2008, Matsumoto 053614 (MBK); Ibid., 3070 m, 30.7.2013, Mu Mu Aung, Ling Shein Mang, Aung Htay \& Har Shin Aung 092798 (MBK); Ibid., 2680-3053 m, 27.8.2013, Fujikawa, Srisanga, Maknoi, Ling Shein Man $\mathcal{E}$ Law Shine 094517 (MBK); Ibid., 2550 m, 1.9.2013, Fujikawa, Srisanga, Maknoi, Tin Mya Soe, Myint Hlaing \& Law Shine 094853 (MBK); Ibid., 2000 m, 14.8.2016, Thet Yu Nwe TY 61 (RAF).

Neottia karoana Szlach., Fragm. Florist. Geobot., Suppl. 3: 117. 1995. Listera micrantha Lindl., J. Proc. Linn. Soc., Bot. 1: 176. 1857.

Type: INDIA, Sikkim, 3050 m, Hooker 353 (Holotype, K-LINDL!).

Terrestrial herbs, autotrophic, stem with two opposite basal leaves. Leaves ovate, shortly petiolate, $0.5-1.1 \times 0.4-0.9 \mathrm{~cm}$, subacute or obtuse. Inflorescences terminal, erect; peduncle slender, 5-10 cm long, minutely pubescent; racemes 3-5 $\mathrm{cm}$ long, pubescent, with 4 to 10 laxly arranged flowers; floral bracts narrowly ovate, c. $1 \mathrm{~mm}$ long, acute. Flowers pale green. Sepals and petals 
similar, elliptic, 2-3.3 × 1-1.5 mm, subacute. Lip rectangular and apically 3-lobed, deflexed, 2-2.5 $\times 0.5-1.7 \mathrm{~mm}$, basally with suborbicular auricles; apical lobes similar, triangular, mid-lobe slightly longer than the side lobes. Column $0.8-1 \mathrm{~mm}$ long. Pedicel and ovary 2-5 $\mathrm{mm}$ long.

Distribution: North-eastern India (Sikkim), Bhutan, northern Myanmar (Kachin State), South-western China (Yunnan).

Specimens examined: MYANMAR, Kachin State: Nogmung Township, 3660 m, 8.1931, KingdonWard 9961 (AMES); Chipwi Township, 3050 m, 7.1919, Kingdon-Ward 3359 (E).

Neottia pinetorum (Lindl.) Szlach., Fragm. Florist. Geobot., Suppl. 3: 118. 1995. Listera pinetorum Lindl., J. Proc. Linn. Soc., Bot. 1: 175. 1857.

Type: INDIA, Sikkim, Lachen Valley, 3050-3350 m, Hooker 355 (Holotype, K-LINDL!).

Terrestrial herbs, autotrophic, with two opposite leaves borne in the middle of the stem. Leaves broadly ovate, sessile, 1-3.5 × 1-4 cm, with cordate or truncate base, acute. Inflorescences terminal, erect; peduncle slender, pubescent; racemes 3-12 $\mathrm{cm}$ long, pubescent, with 2 to 14 laxly arranged flowers; floral bracts ovate-lanceolate, 3-6 $\mathrm{mm}$ long, acuminate. Flowers greenish yellow; sepals and petals not spreading widely. Median sepal narrowly elliptic, 3-4.7 × 2-3 mm, obtuse; lateral sepals elliptic, oblique, 3-4.7 $\times 2-3 \mathrm{~mm}$, acute or obtuse. Petals linear, $3-4 \times 0.5-1 \mathrm{~mm}$, subacute. Lip pendulous, deeply 2-lobed, shape variable, mostly oblong-cuneate or oblanceolate, $7-13 \times 3-5(-7)$ $\mathrm{mm}$; lobes oblong-ovate, $2-5 \times 1-2 \mathrm{~mm}$, obtuse; disk with a longitudinal channel. Column 2-2.5 $\mathrm{mm}$ long; rostellum large. Pedicel and ovary 7-10 mm long.

Distribution: India (North-western Himalayas, Sikkim, Darjeeling), Nepal, Bhutan, Myanmar (Kachin State), South-western and southern China (Xizang, Yunnan, Fujian).

Specimens examined: MYANMAR, Kachin State, Chipwi Township, 2130-2440 m, 4.6.1914, KingdonWard 1631 (E); Ibid., 2135-2440 m, 8.6.1914, KingdonWard 1652 (E).

Nephelaphyllum cordifolium (Lindl.) Blume, Fl. Javae Nov. Ser. 1: t. 61.3. 1858. Cytheris cordifolia Lindl., Gen. Sp. Orchid. Pl.: 129. 1831.

Type: BANGLADESH, 'in montes Sillet', De Silva s.n. in Wallich's Catalogue 3750 (Holotype, K-LINDL!).
Terrestrial herbs with creeping rhizomes, pseudobulbs elongate, $5.5-7 \mathrm{~cm}$ long, one-leaved. Leaf cordate or ovate, prominently petiolate, 5-10 $\times 4-7.5 \mathrm{~cm}$, acute, green with purple mottling. Inflorescences terminal, erect; peduncle $6-7 \mathrm{~cm}$ long, sheathed; racemes 3-4 cm long, with 3 to 6 laxly arranged flowers; floral bracts caducous, linear, $4-7 \times 0.5-1 \mathrm{~mm}$. Flowers: sepals and petals green with purple stripes, lip yellow. Sepals similar, c. $10 \times 2 \mathrm{~mm}$, acute or acuminate. Petals narrowly oblong, about $8 \mathrm{~mm}$ long, acute. Lip 3-lobed, wedge-shaped, c. $11 \times 3.5 \mathrm{~mm}$, spurred; side lobes narrow, diverging; mid-lobe broadly ovate, retuse; disk with three lamellate keels; spur c. $3 \times 2 \mathrm{~mm}$. Column c. $4 \mathrm{~mm}$ long. Pedicel and ovary $7-9 \mathrm{~mm}$ long.

Distribution: North-eastern India, Bangladesh, northern Myanmar (Kachin State).

Specimen examined: MYANMAR, Kachin State, Nogmung Township, 465-560 m, 29.8.1926, Kingdon-Ward 7314 (K).

Oberonia pyrulifera Lindl., Fol. Orchid. 8: 3. 1859.

Type: INDIA, Khasia Hills, Myrung, Griffith s.n. (Holotype, K-LINDL! [barcode K000974232]).

Epiphytic herbs with short stems, 3-5-leaved. Leaves subbasal, laterally compressed, slightly falcate, $2.5-6(-8) \times 0.3-0.7 \mathrm{~cm}$, fleshy, articulate at the base, acuminate. Inflorescences terminal; peduncle subterete, 3-11 cm long, sheathed; racemes $2-8 \mathrm{~cm}$ long, with many subdensely arranged flowers; floral bracts lanceolate, 1.5-2 $\mathrm{mm}$ long, margin erose, acuminate. Flowers yellow. Median sepal ovate-oblong, $0.8-1 \times 0.5-0.6$ $\mathrm{mm}$, obtuse; lateral sepals ovate, $0.8-1 \times 0.6-0.7$ $\mathrm{mm}$, subacute. Petals oblong, $0.8-1 \times 0.3-0.4 \mathrm{~mm}$, obtuse. Lip obovate, deeply 2-lobed, 1.3-1.8 × c. 1 $\mathrm{mm}$, base auriculate; lobes lanceolate, c. $0.6 \times 0.3$ $\mathrm{mm}$, obtuse. Column c. $0.5 \mathrm{~mm}$ long. Pedicel and ovary c. $2 \mathrm{~mm}$ long.

Distribution: Nepal, north-eastern India (Sikkim, Assam, Meghalaya, Manipur), Bhutan, western Myanmar (Chin State), Thailand (Northern, northeastern, South-western), South-western China (Yunnan).

Specimens examined (det. S. Watthana): MYANMAR, Chin State, southern Chin Hills, 2350-2550 m, 7.12.2002, Murata, Tanaka, Sugawara, Nemoto, Iokawa, Shimozono, Hung Maung, Ling Shing Maung \& Cho Cho Win 024737 (MBK); Ibid., 2745 m, 19.11.2011, Ling Shein Man 087843 (MBK); Ibid., 2450-2545 m, 
31.8.2013, Fujikawa, Srisanga, Maknoi, Tin Mya Soe, Myint Hlaing \& Law Shine 094771 (MBK).

Oreorchis oligantha Schltr., Acta Horti Gothob. 1: 152. 1924.

Types: CHINA, Sichuan, Huang-long-si, 21.7.1922, Smith 3257 (Syntype, UPS); Ibid., 23.7.1922, Smith 3658 (Syntype, UPS) [fide Pearce \& Cribb, 1997: 317).

Terrestrial herbs, pseudobulbs ovoid or cylindric, $0.6-1 \times c .0 .5 \mathrm{~cm}$, forming a chain on a short rhizome, one-leaved. Leaf narrowly elliptic, petiolate, $1.8-4$ $\times 0.8-1 \mathrm{~cm}$, acuminate. Inflorescences basal, 8-18 $\mathrm{cm}$ long; peduncle slender, sheathed; racemes $1.5-4 \mathrm{~cm}$ long, with 1 to 4 laxly arranged flowers; floral bracts ovate-deltoid, c. $1 \mathrm{~mm}$ long. Flowers purple, lip white or pale purple and purplespotted. Sepals oblong, 12-15 × 2-2.8 mm, obtuse or subacute; lateral sepals slightly oblique. Petals oblanceolate, $7-9 \times$ c. $2 \mathrm{~mm}$, obtuse or subacute. Lip obovate-oblong, 3-lobed, 8-10 × 5-6 mm, shortly clawed; side lobes oblong-obovate, $1.5-2 \times$ c. $0.5 \mathrm{~mm}$, incurved, obtuse; mid-lobe obovate, $c$. $6 \times 5-6 \mathrm{~mm}$, apically broadly rounded; disk with two lamellae. Column 4.5-6 mm long. Pedicel and ovary 3-5 mm long.

Distribution: Northern Myanmar (Kachin State), South-western and central China (Xizang, Yunnan, Sichuan, Gansu).

Specimens examined: MYANMAR, Kachin State, Nogmung Township, 3960 m, 8.8.1926, KingdonWard $7076(\mathrm{~K})$.

Notes: The specimen Kingdon-Ward 7076 was originally labelled as Galearis sp.

Pogonia yunnanensis Finet, Bull. Soc. Bot. France 44: 419. 1897.

Type: CHINA, Yunnan, west of Tali-fou, between Salouen and Mekong, d'Orleans s.n. (Holotype, P! [barcode P00347670]).

Terrestrial herbs, one-leaved. Leaves elliptic, $1-3(-4.5) \times 0.4-2 \mathrm{~cm}$, base amplexicaul, acute. Inflorescences: peduncle slender; racemes 1-flowered; floral bract borne on the stem up to 2.5 $\mathrm{cm}$ above the leaf, narrowly elliptic or narrowly ovate-lanceolate, 5-17(-20) mm long, acute. Flower not opening widely, light pink with a purple or red lip. Sepals narrowly oblong, $14-17 \times$ c. $3 \mathrm{~mm}$, acute. Petals narrowly oblong, $12-16 \times 3.5-4 \mathrm{~mm}$, obtuse.
Lip narrowly oblong, 3-lobed in upper half, 13-15× c. $3 \mathrm{~mm}$; side lobes narrowly triangular-ovate, 1-2 mm long, acute; mid-lobe linear-oblong, 5-6 × c. 2.5 $\mathrm{mm}$, densely covered with papillose hairs, margin denticulate; disk with two longitudinal keels which become papillose-hairy in the distal part. Column 6-8 mm long. Pedicel and ovary 5-8(-15) $\mathrm{mm}$ long.

Distribution: Northern Myanmar (Kachin State), South-western China (Xizang, Yunnan, Sichuan).

Specimens examined: MYANMAR, Kachin State, Nogmung Township, 3655 m, 8.8.1931, KingdonWard 9766 (BM).

Ponerorchis monantha (Finet) X.H. Jin, Schuit. \& W.T. Jin, Molec. Phylogen. Evol. 77: 51. 2014. Peristylus monanthus Finet, Rev. Gén. Bot. 13: 523. 1901.

Types: CHINA, Sichuan, Tizou, 7.-8.1891, Soulié 315 (Syntype, P!); CHINA, Sichuan, Ta-tsien-lou, 8.1893, Soulié 654 (Syntype, P!; Isosyntype, AMES).

Terrestrial herbs with globose underground root tubers, one-leaved. Leaves basal, lanceolate or oblong, $2-3 \times 0.6-1 \mathrm{~cm}$, acute or obtuse. Inflorescences: peduncle slender, ebracteate; racemes 1-flowered; floral bracts linear-lanceolate, 6-12 mm long, usually longer than ovary, acute. Flower pink, pale purple or white, lip with purple spots. Median sepal erect, ovate, $4-4.5 \times 1.6-1.8$ $\mathrm{mm}$, obtuse; lateral sepals reflexed or spreading, elliptic, $4.5-5 \times$ c. $1.6 \mathrm{~mm}$, subacute or obtuse. Petals ovate, $3.8-4.4 \times 1.6-2 \mathrm{~mm}$, connivent with median sepal, obtuse. Lip ovate-orbicular, deeply 3-lobed, 8-10 × 8-10 mm, shortly clawed, spurred; side lobes oblong-obovate, $4-5 \times 1.8-3 \mathrm{~mm}$, obtuse; mid-lobe broadly obovate, $6-7.5 \times c .7 \mathrm{~mm}$, apically slightly emarginate or deeply notched; disk with fine papillae; spur cylindric, $2.5-4 \mathrm{~mm}$ long, shorter than ovary. Pedicel and ovary 6-11 mm long.

Distribution: Northern Myanmar (Kachin State), South-western and central China (Xizang, Yunnan, Sichuan, Gansu, Shaanxi).

Specimen examined: MYANMAR, Kachin State, Triangle, 3260 m, 27.6.1953, Kingdon-Ward 21604 (AMES).

Zeuxine nervosa (Wall. ex Lindl.) Trimen, J. Ceylon Branch Roy. Asiat. Soc. 9: 90. 1885. Monochilus nervosus Wall. ex Lindl., Gen. Sp. Orchid. Pl.: 487. 1840. 
Types: BANGLADESH, Darwani, 11.3.1809, Buchanan-Hamilton s.n. in Wallich's Catalogue 7381A (Syntype, K-LINDL!); BANGLADESH, Sylhet, De Silva s.n. in Wallich's Catalogue 7381B (Syntype, K-W [fide Pearce \& Cribb, 2002: 113]).

Terrestrial herbs with elongate rhizomes, 3-6-leaved near stem apex. Leaves ovate or elliptic, shortly petiolate, $4-6 \times 1.5-2.5 \mathrm{~cm}$, acute, green with silvery-white blotches or midvein. Inflorescence terminal, $18-28 \mathrm{~cm}$ long; peduncle sheathed, hairy; rachis 3-10 cm long, with a few to over 10 densely arranged flowers; floral bracts ovate-lanceolate, $6-10 \times 3-4.5 \mathrm{~mm}$, puberulent or glabrous, margin ciliate, acuminate. Flowers: sepals brown to yellowish green, petals white, lip white or light yellow; glabrous. Median sepal ovate, $3-5.5 \times 3.5-5 \mathrm{~mm}$, acute or acuminate; lateral sepals oblong-ovate, $4-6.5 \times$ up to $3.5 \mathrm{~mm}$, acute or obtuse. Petals obliquely ovate, $c .5 .5 \times 3.2$ $\mathrm{mm}$, obtuse, laterally connate with median sepal. Lip Y-shaped, 3-partite, c. $7 \mathrm{~mm}$ long; hypochile saccate, c. $2.5 \times 2.5 \mathrm{~mm}$, with two groups of 2-3-digitate calli; mesochile $c$. $1.5 \mathrm{~mm}$ long, with incurved margins; epichile flat, 2-lobed, c. $4 \mathrm{~mm}$ wide, lobes suborbicular, c. $2 \times 2 \mathrm{~mm}$. Column c. $2.5 \mathrm{~mm}$ long; wings large, broadly triangular; rostellum arms c. $1.5 \mathrm{~mm}$ long. Pedicel and ovary 8-9 mm long, glabrous or puberulent.

Distribution: Sri Lanka, India, Nepal, Bhutan, Bangladesh, Myanmar (Kayin State), Andaman Islands, Thailand, Laos, Vietnam, Cambodia, south-western China (Yunnan), Taiwan, southern Japan (Nansei-shoto), Philippines.

Specimens examined: MYANMAR, Kayin State, Hpa-an or Myawaddy District, 3.1904, Burkill 24458 p.p. (K).

\section{Acknowledgements}

We thank the curators of AMES, BM, E, K, MBK, NY, RAF and SING for providing access to their collections, and Bhakta Bahadur Raskoti and Santi Watthana for help with identifications. Long-time collaborator Saw Lwin (Myanmar Floriculturist Association) is thanked for providing specimens for identification. The first author is also indebted to the Myanmar Forest Department for issuing collecting permits, and to the National Parks Board, Singapore, for financial support.

\section{Literature Cited}

Barretto, G., Cribb, P. \& S. Gale 2011. The Wild Orchids of Hong Kong. Natural History Publications, Kota Kinabalu; in association with Kadoorie Farm and Botanic Garden, Hong Kong.

Bhattacharjee, A. \& R.K. Bhakat 2010. Lectotypifications in Goodyera R. Br. (Orchidaceae). Rheedea 20(2): 73-75.

Bhattacharjee, A. \& H.J. Chowdhery 2012. Notes on two species of Goodyera (Orchidaceae). Kew Bull. 67: 503-510.

Chen, S.C., Liu, Z.J., Zhu, G.H., Lang, K.Y., Tsi, Z.H., Luo, Y.B., Jin, X.H., Cribb, P.J., Wood, J.J., Gale, S.W., Ormerod, P., Vermeulen, J.J., Wood, H.P., Clayton, D. \& A. Bell 2009. Orchidaceae. In: Wu, Z.Y., Raven, P.H. \& D.Y. Hong (Eds.), Flora of China. Vol. 25. Science Press, Beijing \& Missouri Botanical Garden Press, St. Louis. pp. 1-570.

Kumar, P., Gale, S.W., Pedersen, H.Æ., Phaxaysombath, T., Bouamanivong, S. \& G.A. Fischer 2018. Additions to the orchid flora of Laos and taxonomic notes on orchids of the Indo-Burma region. Taiwania 63(1): 61-83.

Liu, Q., Zhou, S.S., Jin, X.H., Pan, B., Kyaw Win Maung, Myint Zyaw, Li, R., Quan, R.C. \& Y.H. Tan 2018. Dendrobium naungmungense (Orchidaceae, Dendrobieae), a new species from Kachin State, Myanmar. PhytoKeys 94: 31-38.

Nuammee, A., Seelanan, T., Suddee, S. \& H.A. Pedersen 2016. Notes on Crepidium (Orchidaceae): two new combinations, a putative natural hybrid, and four species newly recorded for Thailand. Thai Forest Bull., Bot. 44(1): 35-44.

O'Byrne, P. 2012. A revision of Cleisostoma in Peninsular Malaysia. Malesian Orchid J. 9: 57-114.

Pearce, N.R. \& P.J. Cribb 1997. A revision of the genus Oreorchis (Orchidaceae). Edinburgh J. Bot. 54(3): 289-328.

Pearce, N.R. \& P.J. Cribb 2002. The Orchids of Bhutan. In: Flora of Bhutan. Vol. 3(3). Royal Botanic Garden Edinburgh, UK, and Royal Government of Bhutan. 
Raskoti, B.B., Schuiteman, A., Jin, W.T. \& X.H. Jin 2017. A taxonomic revision of Herminium L. (Orchidoideae, Orchidaceae). PhytoKeys 79: $1-74$.

Seidenfaden, G. 1973. Notes on Cirrhopetalum Lindl. Dansk Bot. Ark. 29(1): 7-260.

Seidenfaden, G. 1975. Orchid genera in Thailand I. Calanthe R. Br. Dansk Bot. Ark. 29(2): 1-50.

Seidenfaden, G. 1976. Orchid genera in Thailand IV. Liparis L.C. Rich. Dansk Bot. Ark. 31(1): $1-105$.

Seidenfaden, G. 1977. Orchid genera in Thailand V. Orchidoideae. Dansk Bot. Ark. 31(3): 1-149.
Seidenfaden, G. 1978. Orchid genera in Thailand VII. Oberonia Lindl. and Malaxis Sol. ex Sw. Dansk Bot. Ark. 32(1): 6-94.

Seidenfaden, G. 1979. Orchid genera in Thailand VIII. Bulbophyllum Thouars. Dansk Bot. Ark. 33(3): 1-228.

van der Burgh, W. \& E.F. de Vogel 1997. Revision of the orchid genera Chrysoglossum, Collabium, Diglyphosa and Pilophyllum (Subtribe Collabiinae). Orchid Monogr. 8: 135-174.

Received: 04.04.2018

Revised and Accepted: 31.05.2018 